\title{
Modeling and information structures for supervisory control of flexible manufacturing systems
}

\author{
D. Labalo, A. Gurel, F. L. Lewis,
} Automation and Robotics Research Institute, The University of Texas at Arlington, 7300 Jack Newell Blvd. S, Ft. Worth, Texas 76118-7115, tel. 817-272-5972, fax. 817-272-5952, email flewis@controls.uta.edu.

\section{S. Bogdan,}

Faculty of Electr. Eng. and Comp., University of Zagreb, Unska 3, HR-10000 Zagreb, Croatia.

\begin{abstract}
In this paper we discuss a new matrix-based modeling technique for flexible manufacturing systems (FMS). Four Task Plan Matrices can completely describe the job sequencing and resource assignment details of an FMS. The matrices come directly from plans generated by machine planners, or from the bill of materials and resource requirements matrix. Given the matrices, a rule-based supervisory controller operates in real-time to sequence jobs and assign resources depending on dynamic situational information about parts available, resources idle, and jobs completed. The function of the FMS can be quickly changed when new products are needed simply by changing the four matrices. By deriving explicit mathematical formulas for $\mathrm{p}$-invariants and the resource requirement matrix for generalized reentrant flow lines, NP-complexity problems are avoided, which is often an issue in other modeling techniques.
\end{abstract}

Keywords

Manufacturing, discrete event systems, flexible manufacturing systems. 
For complex manufacturing processes it is very difficult to provide a mathematical model suitable for fast redesign of dispatching and routing controllers. In existing models (e.g. Petri nets) the functions of the workcell and of the rule-based controller are not separated, so that fast reconfiguration for changing goals and new products is not easy. Dealing with machine failures, changes in product mix, technological changes, changes in goals, etc., requires modifying the controller rules in real-time, which is difficult.

In Industrial Engineering there is a wide variety of techniques available for manufacturing scheduling such as the assembly tree [Wolter et al. 1992], bill of material (BOM) [Elsayed and Boucher 1994], resource requirement (RR) matrix [Kusiak 1992], and sequencing matrix [Steward 1981, Warfield 1973]. Unfortunately, these are treated as heuristic aids and cannot provide a rigorous mathematical framework as needed for computer analysis and control of manufacturing systems. Industrial Engineering approaches such as queuing methods [Kumar 1993], Operations Research (OR) approaches [Conway 1967, Graves 1984], etc., can provide rigorous analysis and are convenient for determining steady-state solutions. Transient analysis and analysis for large-scale systems is difficult.

Rigorous approaches for analysis of discrete event dynamical systems (DEDS) are emerging in Electrical Engineering and Computer Engineering. DEDS include communication networks, manufacturing systems, Intelligent Vehicle and Highway Systems, etc. Available analysis tools include Petri nets (PN) [Desrochers 1990, Krogh, 1991, Murata 1989, Peterson 1981, Zhou 1993], the max/plus algebra [Cohen 1985], perturbation analysis [Ho 1987, 1989], object oriented techniques [Glassey 1990], etc. Unfortunately, it is difficult to provide a mathematical model for a manufacturing system that can be used to guarantee deadlock-free performance, efficient shared-resource conflict resolution, and suitable part-routings. PN are difficult to construct. The max/plus equations cannot be written down unless a decision-free PN is available. Finally, computational complexity associated with these techniques very often leads to intractability [Garey and Johnson 1979].

This paper discusses an analytic matrix framework for discrete event manufacturing systems modeling, analysis and control. By taking advantage of this matrix model, explicit formulae are provided for some useful information structures used in analysis and design, thereby correcting the prime deficiency of Petri net and other modeling concepts - the inability to provide efficient computational techniques.

\section{INTELLIGENT MANUFACTURING}

With the development of automation technology its supporting systems - planning, scheduling, and control - have gained importance. Fast response to market demands and flexibility involves sophisticated control architectures in 
manufacturing systems. The hybrid nature of FMS and flexibility issues increase the role of multi-layer architectures with enhanced sensing, modeling, and execution capabilities. All of these concepts are captured by the so-called intelligent control (IC) architectures that have been studied extensively in the last decade [Antsaklis and Passino 1992, Kusiak 1992, Saridis 1996].
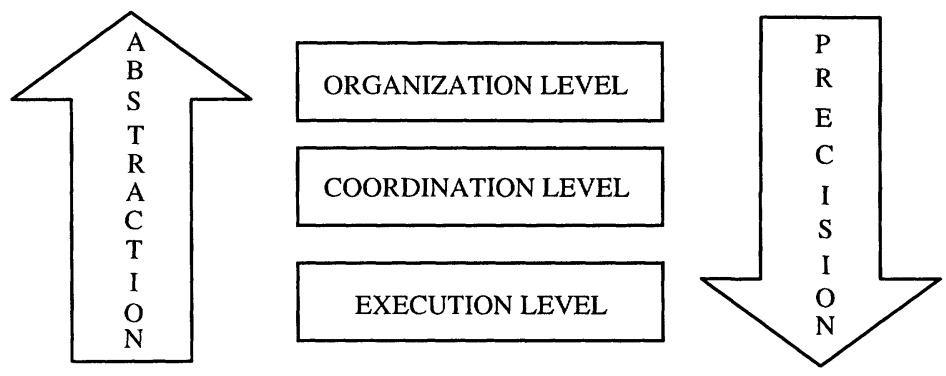

Figure 1 Three-level intelligent control architecture.

A general IC architecture based on work by Saridis is given in Figure 1, which illustrates the principle of decreasing precision with increasing abstraction. In this figure, the organization level is considered to be the highest level of decisionmaking where the Production Plan (PP), Master Production Schedule (MPS), and Material Requirements Planning (MRP) is developed through information provided by the marketing department forecasts and upper management plans. This level has a role of a manager that schedules and assigns tasks, performs task decomposition and planning, and determines for each task a required partial ordering of jobs. The coordination level performs the exact job sequencing, coordinating the workcell agents or resources. In addition, in the case of shared resources it must execute dispatching and conflict-resolution decisions. The agents or resources might include automated machine tools, robot manipulators, material handling, storage systems and computer hardware for planning, data acquisition and decision-making. The execution level contains a closed-loop controller for each agent that is responsible for its real-time performance.

It is shown [Harris et al. 1997] that a task plan produced in the organization level is equivalent to four matrices; two of them capturing the information on job sequencing and two others describing the assignment and release of resources that are needed to perform the jobs. The matrices are passed to a supervisory controller in the Coordination level for real-time execution of the plan.

Based upon the matrix model of discrete event systems [Lewis et al.1993] it is easy to design the intelligent rule-based workcell controller. The objective of designing the controller is to provide two main functions: (1) job sequencing, (2) resource allocation and conflict resolution. The controller design is based upon a matrix model of discrete manufacturing systems that is fully consistent with the IC 
architecture described above. In the next section, the matrix model of discrete event manufacturing systems [DEMS] will be discussed in more detail.

\section{MATRIX MODEL FOR DISCRETE EVENT MANUFACTURING SYSTEMS}

A new rule-based DEMS matrix model [Lewis et al. 1993] is now described that provides a framework for rigorous analysis of discrete event manufacturing systems. The matrix discrete model is described by the following equations:

Logical matrix state equation

$\bar{x}=\mathrm{F}_{\mathrm{v}} \bar{v}_{\mathrm{c}}+\mathrm{F}_{\mathrm{r}} \overline{\mathrm{c}}_{\mathrm{c}}+\mathrm{F}_{\mathrm{u}} \bar{u}+\mathrm{F}_{\mathrm{D}} \bar{u}_{\mathrm{D}}$.

Job start equation

$v_{\mathrm{s}}=\mathrm{S}_{\mathrm{v}} x$.

Resource release equation

$r_{\mathrm{s}}=\mathrm{S}_{\mathrm{r}} x$.

Product output equation

$y=\mathrm{S}_{\mathrm{y}} x$

PN transition equation

$m(\mathrm{k}+1)=m(\mathrm{k})+\mathrm{W}^{\mathrm{T}} x(\mathrm{k}+1)$.

The matrix state equation is a set of rules, so that it is formally a rule base. Entries of 1 in $\mathbf{v}_{\mathrm{c}}$ and $\mathbf{r}_{\mathrm{c}}$ respectively indicate jobs complete and resources idle. A computed entry $x_{i}=1$ of logical state vector $\mathbf{x}$ indicates that all the conditions required to fire rule $i$ are met. Entries of 1 in $\mathbf{v}_{s}, \mathbf{r}_{s}$ respectively indicate the jobs to be started or resources to be released. The overbar in equation (1) denotes logical negation. (Given a natural number vector $a$, its negation $\bar{a}$ is such that $\bar{a}(\mathrm{i})=0$ if $a(i)>0$, and 1 otherwise; for instance, job complete is shown by 0 . Overbar notation is used similarly throughout the paper). Finally, all matrix operations are defined to be in and/or algebra where multiplication is replaced by 'and' and addition by 'or'.

These equations are easy to write down: $F_{\mathrm{v}}$ is Steward's job sequencing matrix (JSM) [Steward 1962], and can be determined from the Bill of Materials (BOM) [Elsayed and Boucher, 1994] or the assembly tree [Chakrabarty and Tsao, 1992]. 
$F_{v}(i, j)=1$ if job $j$ is required as an immediate precursor to job $i$ (equivalently, in the $\mathrm{BOM}$, if subassembly $\mathrm{j}$ is required to produce subassembly $i$ ). $\mathrm{F}_{\mathrm{r}}$ is the resource requirements matrix of Kusiak (1992), which is assigned by the shop-floor engineer, with $F_{r}(i, j)=1$ if resource $j$ is required for job $i$. The job sequencing matrix $F_{v}$ and the resource requirements matrix $F_{r}$ have long been used as heuristic design aids by industrial engineers, with some possibility for limited analysis (as discussed e.g. by Warfield (1973) in the case of $F_{v}$ and Kusiak (1992) in the case of $\left.F_{r}\right)$. The logical matrix model elevates these design tools to formal computation elements.

The job start matrix $S_{v}$ and the resource release matrix $S_{r}$ are additional matrices that must be introduced to complete the description of the controller, and they are equally direct to write down for a specific DEMS. $S_{v}(i, j)=1$ if job $i$ is to be started when all the requirements of logical component $x_{j}$ of the decision rule vector $\mathbf{x}$ are satisfied (i.e., $x_{j}$ is set high). When there are no routings in the system this matrix has a diagonal of 1's. In the more general job shop case, it has multiple ones in columns, which correspond to job routing decisions. $S_{r}(i, j)=1$ if resource $i$ to be released when logical state component $\mathrm{x}_{\mathrm{j}}$ is set high.

Input $\mathbf{u}$ and output $\mathbf{y}$ represents, respectively, raw parts entering and finished products leaving the cell. Input $\mathbf{u}_{\mathrm{D}}$ is a conflict resolution input that selects the jobs to be initiated when there are simultaneous requests involving shared resources, a situation which may occur when the resource requirements matrix $F_{r}$ has multiple 1 's in the same column. To resolve such potential conflict and turn the controller into a 'decision-free' graph [Cofer and Garg, 1992], it is therefore necessary to add the extra dispatching control input $\mathbf{u}_{\mathrm{D}}$, which is allowed to have only one of its entries of a given column high at any given instant.

It is exactly in the selection of the dispatching control input $\mathbf{u}_{\mathrm{D}}$ that the dispatching rules available in the industrial engineering literature [Panwalker and Iskander 1977; Elsayed and Boucher 1994] can be used.

It is important to label the jobs causally in the formulation of the controller so as to obtain $F_{v}$ and $S_{v}$ in lower triangular form [Warfield 1977]. A causal ordering is important also in proving the results on the computation of deadlock analysis structures. The components of the rule vector $\mathbf{x}$ should then be ordered in accordance with the jobs they initiate. This procedure corresponds to numbering the jobs from bottom to top in an assembly tree.

The transition equation (5) together with matrix state equation (1) provide complete dynamical description of the system where

$$
m(k)=\left[\begin{array}{l}
v_{c}(k) \\
r_{c}(k)
\end{array}\right],
$$

represents the PN marking vector at discrete event iteration $k$, and

$$
W=\left[\begin{array}{ll}
S_{v}{ }^{T}-F_{v} & S_{r}{ }^{T}-F_{r}
\end{array}\right],
$$

represents the PN incidence matrix. 
Control of discrete event manufacturing systems (DEMS) [Lewis et al. 1993] is examined from a system theory perspective which makes an explicit distinction between the plant or workcell (comprised of machines and resources to be controlled) and the controller (representing a real-time decision-making scheduler in the coordination level which implements a rule base). This rigorous point of view is able to provide a design algorithm for discrete event control systems that afford a very convenient approach to the design of DEMS. The controller has an intelligent rule-based structure and consists of inner loops, where no conflict resolution is needed and outer loops, where logic is needed to resolve sharedresource conflicts. The DEMS matrix controller design will be illustrated through the example.

Figure 2a depicts an assembly tree, which shows the required sequence of actions (jobs) to produce a product. This assembly tree contains information analogous to the BOM; it does not include any resource information.

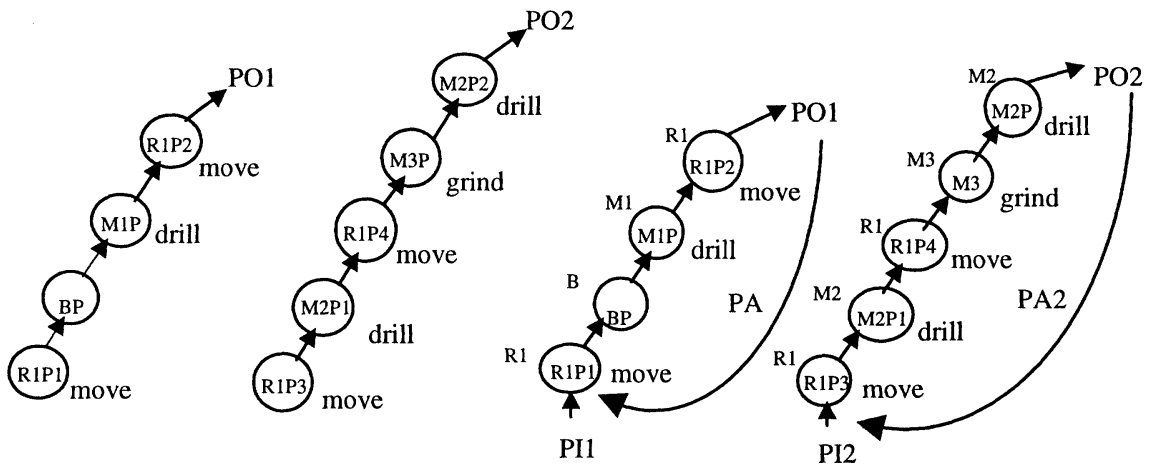

Figure 2a Assembly tree.

Figure 2b Subassembly tree.

To build a dispatching controller for shop-floor installation to perform this particular assembly task, the resources available must be added. Figure $2 \mathrm{~b}$ shows a subassembly tree for the assembly task, which includes the resource requirement information.

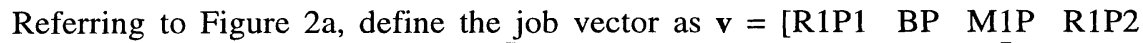
R1P3 M2P1 R1P4 M3P M2P2 $]^{\mathrm{T}}$, the input vector $\mathbf{u}=\left[\begin{array}{ll}\mathrm{PI} 1 & \mathrm{PU} 2\end{array}\right]^{\mathrm{T}}$, and the output vector $\mathbf{y}=\left[\begin{array}{ll}\mathrm{PO} 1 & \mathrm{PO} 2\end{array}\right]$. The job vector $\mathbf{v}$ has two interpretations: as a status output of the workcell, it denotes completed jobs; in this role it is denoted as $\mathbf{v}_{\mathrm{c}}$. On the other hand, as an input of the workcell, it represents the job start command vector; in which case it is denoted by $\mathbf{v}_{\mathbf{s}^{*}}$. Define the controller state vector $\mathbf{x}$, each 
component corresponding to a unique component of vectors $\mathbf{v}$ or $\mathbf{y}$, thus representing the initiation rule for the latter. Then, the job start equation and the part output equations are:

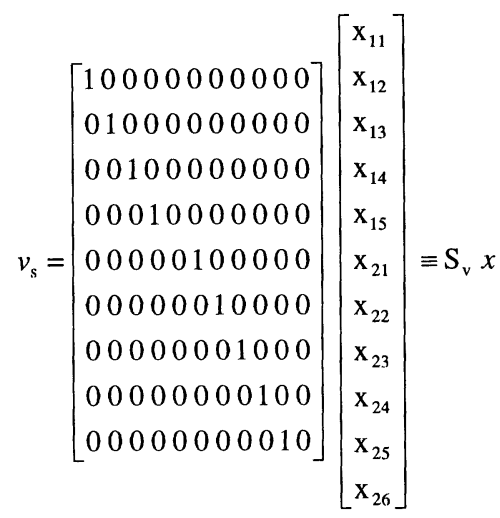

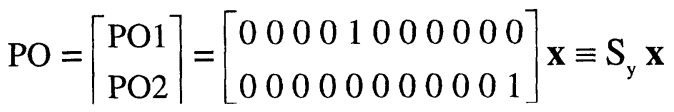

Referring to Figure $2 \mathrm{~b}$, define the resource vector $\mathbf{r}$ as $\mathbf{r}=\left[\begin{array}{lll}\mathrm{M} 1 \mathrm{~A} & \mathrm{M} 2 \mathrm{~A} & \mathrm{M} 3 \mathrm{~A}\end{array}\right.$ BA R1A PA1A PA2A ] $]^{T}$, where ' $A$ ' stands for 'available'. Vector $\mathbf{r}$ accounts for all the resources and pallets in the flowline. Now, by inspection of the subassembly tree, write down $\mathrm{F}_{\mathrm{v}}, \mathrm{F}_{\mathrm{r}}$ and $\mathrm{F}_{\mathrm{u}}$, and obtain the controller state equation

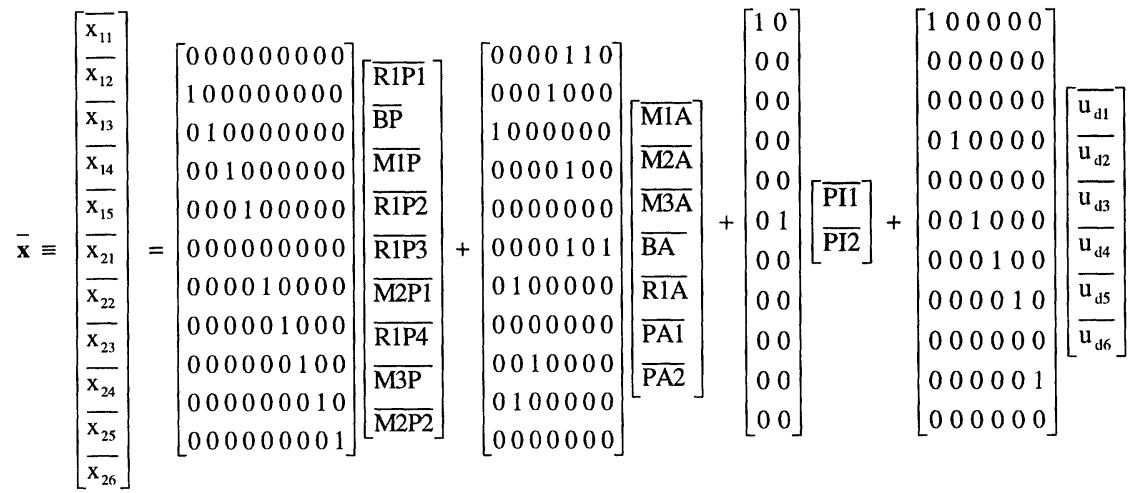

In this equation, row 7, for instance, indicates that component $\mathrm{x}_{22}$ is set high if job R1P3 is done and resource M2 is available. To verify this one can formally work out the logical operations using deMorgan's rules. 
The last issue to be resolved in this design is that of resource and pallet release. Thus, using industrial engineering experience and Figure $2 b$, obtain the resource and pallet release matrix $S_{r}$ and write down the resource release equation

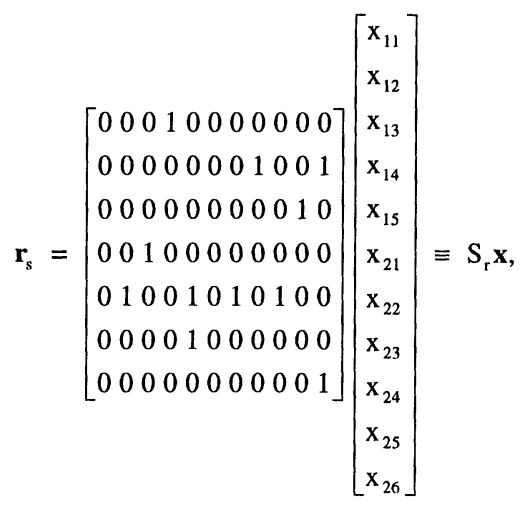

where subscript 's' denotes a command to the workcell to start resource release.

It should be emphasized that $F_{v}$ and $S_{v}$ depend only on job sequencing information, while all the resource information is contained in $F_{r}$ and $S_{r}$. Note that rows containing multiple ones in $\mathrm{S}_{\mathrm{r}}$ correspond to columns having multiple ones in $F_{r}$. The dispatching input, $\mathbf{u}_{\mathrm{D}}$, is needed as a control decision input, since $\mathrm{F}_{\mathrm{r}}$ has multiple 1 's in its $2^{\text {nd }}$ a and $6^{\text {th }}$ columns, indicating that machine M2 and robot R1 are shared resources.

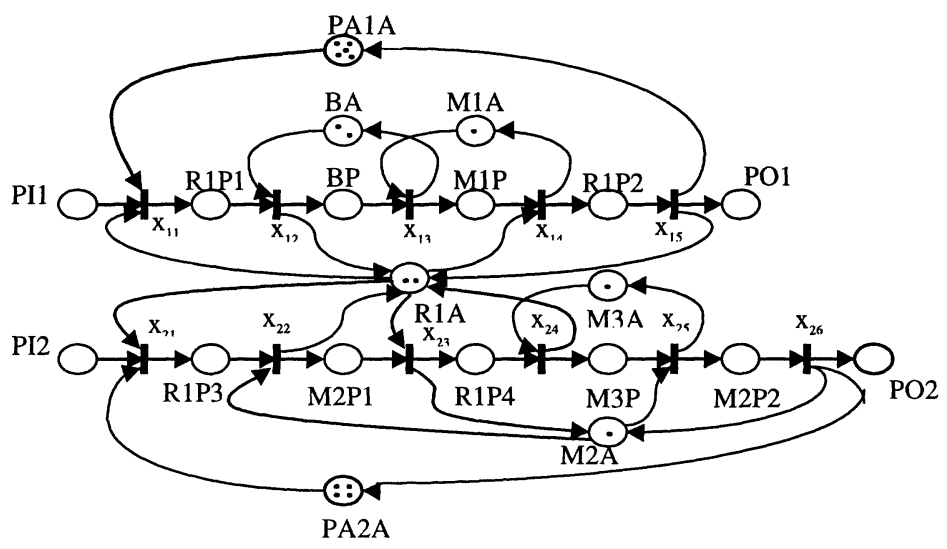

Figure 3 Petri net representation of the workcell with shared resources.

It is straightforward to derive the Petri net description of a manufacturing system from the matrix model equations (1)-(4). This allows all the Petri net analyses tools to be used for DEMS within the matrix model framework. Figure 3, depicts the 
Petri net model with an initial marking reflecting the number of resources available in the workcell (system idle).

DEMS structure analysis and associated computational complexity issues are addressed in the next section. Taking advantage of the matrix model, explicit formulas are provided for some useful information structures used in analysis and design, thereby correcting the prime deficiency of Petri net and other modeling concepts - the inability to provide efficient computational techniques.

\section{MATRIX FRAMEWORK AND COMPUTATIONAL COMPLEXITY}

There are many distinct analysis and design problems to be solved in FMS, including scheduling with optimality, computation of PN p-invariants to determine resource loops, analysis of deadlocks and circular waits, design and implementation of deadlock avoidance strategies, and design/selection of dispatching and routing algorithms. These problems have varying degrees of complexity, which depends on whether one has a flow line, assembly line, or general job shop structure.

The theory of NP-completeness [Garey and Johnson 1979] potentially provides a comprehensive approach for analysis of computational complexity in FMS. Many traditional scheduling and sequencing problems have been found to be in NP so that significant increase in computing power do not significantly improve computational capabilities. Thus it has been necessary to develop heuristics or approximate methods for analysis and solution.

Petri Nets have been extensively used in the analysis of manufacturing systems with quite variable results. The $\mathrm{PN}$ incidence matrix has been used to study structural properties of FMS, including determination of the siphons [Boer 1994] and deadlock avoidance [Lewis et al. 1996]. However PN applications have not fully exploited the matrix nature of the system structure and lead to computational intractability.

The new DE matrix model overcomes one of the prime deficiencies of PN theory - it provides rigorous computational techniques for DE systems. As an example we select the representative problem of determining the p-invariants for the very general class of reentrant flow lines with assembly that allow more than one resource to be assigned to job, as well as to assign a resource to more than one job in the sequence. The p-invariants have been extensively studied in work by [Desrochers 1990], [Jeng and DiCesare 1993], [Zhou 1993] and elsewhere. They provide the basis for several FMS control techniques that involve dispatching of shared resources. The Matrix model allows p-invariants to be given by an explicit matrix formula. 
Using Petri net terminology, a p-invariant can be defined as a place vector $\mathbf{p}$ having elements of zeros and ones that is in the nullspace of incidence matrix $\mathrm{W}$, that is

$$
W \cdot p=\underline{0} .
$$

Where $W$ is given by (7) and place vector can be partitioned by vector of job places $v$ and vector of resource places $r$. Now (12) becomes

$$
\left[\begin{array}{ll}
W_{v} & W_{r}
\end{array}\right]\left[\begin{array}{l}
v \\
r
\end{array}\right]=\underline{0},
$$

which is equivalent with

$$
W_{v} \cdot v=-W_{r} \cdot r
$$

To find $v$ vectors, multiply (12) by $W_{v}^{T}$ to get the square term on the left side and than take its inverse getting

$$
v=-\left(W_{v}^{T} \cdot W_{v}\right)^{-1} \cdot W_{r} \cdot r
$$

Now for each particular resource, set the $r$ vector by putting the 1 in appropriate row and vector $v$ will give us the set of jobs contained in the corresponding resource loop. Make note that $v$ vectors form a resource requirement matrix (RR) [Kusiak 1992] that provides the basis for dispatching of shared resources (MDR rule). To obtain the (RR) matrix set $r=I$, the identity resulting in

$$
F_{k}=-\left(W_{v}^{T} \cdot W_{v}\right)^{-1} \cdot W_{r}
$$

Every conservative system (very broad class of manufacturing systems have this property) has one and only one set of jobs that together with corresponding resource form a p-invariant vector. Therefore in these systems, matrix $\left(W_{v}{ }^{T} \cdot W_{v}\right)$ is always invertible.

The complete set of p-invariants is given by the columns of the matrix,

$$
P=\left[\begin{array}{c}
F_{k} \\
I
\end{array}\right],
$$

where $F_{k}$ is the resource requirement matrix, and $I$, the identity matrix.

To illustrate the formula (17), consider the example in Figure 4.This example belongs to the very general class of reentrant flow lines with assembly that allow more than one resource to be assigned to job, as well as to assign a resource to more than one job in the sequence. 


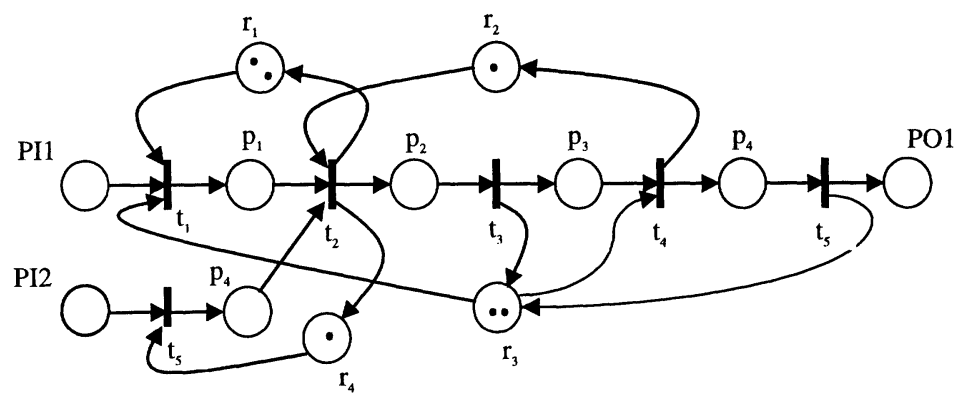

Figure 4 Example of generalized reentrant flow line with assembly.

From (7),

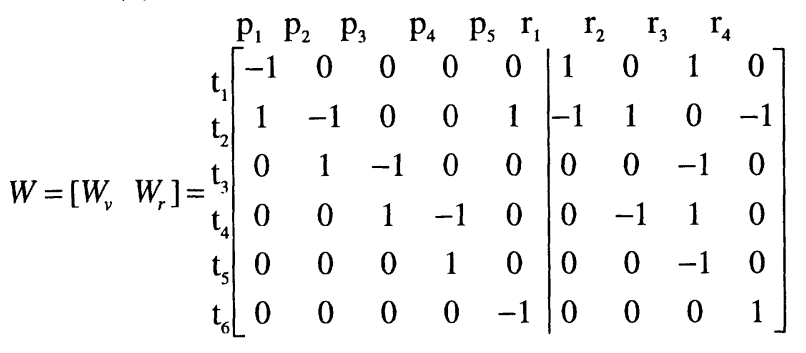

Employing now (17) one obtains p-invariants as the columns of:

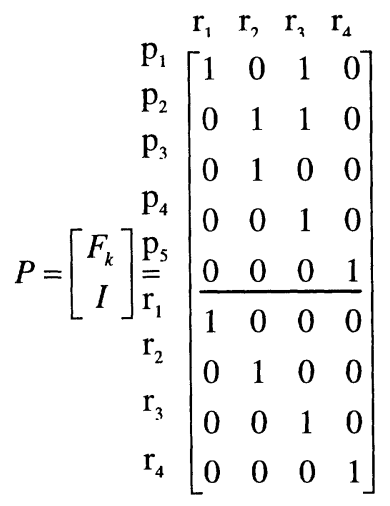

Using the formulas (16) and (17) allows one to compute mathematically pinvariants and resource requirement matrix for generalized class of flow lines where it is very difficult to obtain any results by inspection. 
A new rule-based DEMS matrix model is adopted that provide a framework for rigorous analysis and control design of discrete event manufacturing systems. The matrices in the matrix model description come directly from industrial engineering tools such as the bill-of-materials, assembly tree, and resource requirements matrix. By deriving explicit mathematical formulas for p-invariants and the resource requirement matrix for generalized reentrant flow lines, NP-complexity problems are avoided, which is often an issue in other modeling techniques.

\section{REFERENCES}

R. Akella, "Special Issue on Manufacturing", IEEE Trans. Robotics and Automation, vol. 6, no. 6, Dec. 1990.

P.J. Antsaklis and K.M. Passino. An Introduction to Intelligent and Autonomous Control. Kluwer, Boston, MA, 1992.

Z.A. Banaszak and B.H. Krogh, "Deadlock avoidance in flexible manufacturing systems with concurrently competing process flows," IEEE Trans. Robotics and Automation, vol. 6, no. 6, pp. 724-734, Dec. 1990.

E.R. Boer and T. Murata, "Generating basis siphons and traps of Petri nets using the sign incidence matrix," IEEE Trans. Circuits and Systems, vol. 41, no. 4, pp. 266-271, April 1994.

T.B. Boffey, (1982), Graph Theory in Operations Research, Macmillan, London.

X. Cao and Y.C. Ho, "Models of discrete event dynamic systems," Control Systems Magazine, vol. 10, no. 4, pp 69-76, 1990.

G. Cohen, D. Dubois, J.P. Quadrat, and M.Viot, "A linear-system-theoretic view of discrete-event processes and its use for performance evaluation in manufacturing," IEEE Trans. Automat. Control, vol. AC-30, no.3, pp. 210220, Mar. 1985.

R.W. Conway, W.L. Maxwell, and L.W. Miller, Theory of Scheduling. Reading, MA, Addison-Wesley, 1967.

C.L.P. Chen and C. Wichman, "A CLIPS rule-based planning system for mechanical assembly," Proc. NSF DMS Conf., pp. 837- 841, Atlanta, 1992.

H.M. Deitel, An Introduction to Operating Systems, chap. 6, Addison-Wesley, Reading, MA, 1984.

A.A. Desrochers, Modeling and Control of Automated Manufacturing Systems, IEEE Computer Society Press, 1990.

E.A. Elsayed and T.O. Boucher, Analysis and Control of Production Systems, 2nd ed., Prentice-Hall, Englewood Cliffs, NJ, 1994.

S.D. Ezpeleta, J.M. Colom, and J. Martinez, “A Petri net based deadlock prevention policy for flexible manufacturing systems," IEEE Trans. Robotics and Automation, vol. 11, no. 2, pp. 173-184, Apr. 1995.

M.R. Garey, and D.S. Johnson, Computers and Intractability: a Guide to the Theory of NP-completeness. Freeman, San Francisco, CA, 1979.

C.R. Glassey and M.G.C Resende, "A scheduling rule for job release in semiconductor fabrication," Operations Research Letters, vol. 7, pp. 213-217, 1988. 
S.C. Graves, "A review of Production Scheduling", Operation Research, vol. 29, pp- 646-675, 1984.

D. Gracanin, P. Srinivasan, K. Valavanis, "Parametrized Petri nets: properties and applications to automated manufacturing systems," Proc. IEEE Mediterranean Symp. New Directions in Control and Automation, pp. 48- 55, June 1994.

A Gurel, O.C. Pastravanu, and F.L. Lewis, "A robust approach in deadlock-free and live FMS design," Proc. IEEE Mediterranean Symp. New Directions in Control and Automation, pp. 40-47, June 1994.

B. Harris, F.L. Lewis and D. J. Cook, "Machine Planning for Manufacturing: Dynamic Resource Allocation and On-Line Supervisory Control," to appear in the Journal of Intelligent Manufacturing

Y.C. Ho, “Dynamics of discrete event systems," Proc. IEEE. pp. 3-6\}, Jan. 1989.

Y.C. Ho and X. Cao, Perturbation Analysis of Discrete Event Systems, Kluwer, Boston, 1991.

F.-S. Hsieh and S.-C. Chang, "Dispatching-driven deadlock avoidance controller synthesis for flexible manufacturing systems," IEEE Trans. Robotics and Automation, vol. 10, no. 2, pp. 196-209, April 1994.

M.D. Jeng and F. DiCesare, "A synthesis method for Petri net modeling of automated manufacturing systems with shared resources," Proc. IEEE Conf. Decision and Control, pp. 1184- Dec. 1992.

E. Kasturia, F. DiCesare, A. Desrochers, "'Real time control of multilevel manufacturing systems using colored petri nets," Proc. IEEE Conf. Robotics Automat., pp. 1114-1119, 1988.

M. Klein, "Supporting conflict resolution in cooperative design system," IEEE Trans. Sys., Man, and Cybernetics, vol.21, no. 6, pp. 1379-1390, Nov./Dec. 1991.

T.K. Kumaran, W. Chang, N. Cho, R.A. Wysk, (1994), “'A structured approach to deadlock detection, avoidance, and resolution in flexible manufacturing systems," Int. J. Prod. Res, vol. 32, no. 10, pp. 2361-2379.

P.R Kumar and S.P. Meyn, "Stability of queueing networks and scheduling policies," IEEE Trans. Automat. Control, vol. 40, no. 2, pp. 251-260, Feb. 1995.

A. Kusiak, "Intelligent scheduling of automated machining systems," in Intelligent Design and Manufacturing, ed. A. Kusiak, New York: Wiley, 1992.

K. Lauterbach, "Linear algebraic calculation of deadlocks and traps," Concurrency and Nets, ed. K. Voss and H.J. Genrich, Springer-Verlag, 1987.

F.L. Lewis, O.C. Pastrovanu and H-H. Huang, "Controller design and conflict resolution for discrete event manufacturing systems," Proc. IEEE Conf. Decision and Control, pp. 32883293, San Antonio, Dec. 1993.

F.L. Lewis, A.Gurel, S.Bogdan, A. Doganalp, O. Pastrovanu, "Analysis of Deadlocks and Circular Waits Using," submitted 1996.

S.H. Lu and P.R. Kumar, "Distributed scheduling based on due dates and buffer priorities," IEEE Trans. Automat. Control, vol. 36, no. 12, pp. Dec. 1991.

P.B. Luh and D.J. Hoitomt, "Scheduling of manufacturing systems using the Lagrangian relaxation technique," IEEE Trans. Automat. Control, vol. 38, no. 7, July 1993. 
T. Murata, "Petri nets: properties, analysis and applications," Proc. IEEE, vol. 77, no. 4, pp. 541-580, Apr. 1989.

T. Murata, N. Komoda, K. Matsumoto, and K. Haruna, 'A Petri net-based controller for flexible and maintainable sequence control and its applications in factory automation," IEEE Trans. Ind. Electronics, vol. IE-33, no. Feb. 1986.

Panwalker and W. Iskander, "'A survey of scheduling rules," Operations Research, vol. 26, no. 1, pp. 45-61, Jan.-Feb. 1977.

Peterson, J.L. (1981), Petri Net Theory and the Modeling of Systems, Prentice-Hall, Englewood Cliffs, NJ.

P.J. Ramadge and W.M. Wonham, "'The control of discrete event systems," Proc. IEEE, vol. 77, pp. 81-98, 1989.

G.N. Saridis. Arhitectures for Intelligent Control. Intelligent Control Systems, IEEE Press 1996.

D. V. Steward, “On an approach to techniques for the analysis of the structure of large systems of equations," SIAM Review, vol. 4, no. 4, Oct. 1962.

D. V. Steward, "The Design Structure System: A Method for Managing the Design of Complex Systems," IEEE Trans. Engineering Management, pp. 7174, August 1981.

N. Viswanadham, Y. Narahari, and T.L. Johnson, "Deadlock prevention and deadlock avoidance in flexible manufacturing systems using Petri net models," IEEE Trans. Robotics and Automation, vol. 6, no. 6, pp. 713-723, Dec. 1990.

Warfield, "Binary matrices in system modeling," IEEE Trans. Systems, Man, Cybern, vol. SMC-3, no. 5, pp. 441-449, Sept. 1973.

Wolter, S. Chakrabarty, and J. Tsao, "Methods of knowledge representation for assembly planning," Proc. NSF Design and Manuf. Sys. Conf, pp. 463-468, Jan. 1992.

Wysk, N.S. Yang, and S. Joshi, “Detection of deadlocks in flexible manufacturing cells," IEEE Trans. Robotics and Automation, vol. 7, no. 1991.

M.C. Zhou, F. DiCesare, A.D. Desrochers, "A hybrid methodology for synthesis of Petri net models for manufacturing systems," IEEE Trans. Robotics and Automation, vol. 8, no. 3, pp. 350-361, Jun. 1992.

M.C. Zhou and F. DiCesare, Petri Net Synthesis for Discrete Event Control of Manufacturing Systems, Boston: Kluwer, 1993.

Dragan Labalo is a graduate research assistant at the Automation \& Robotics Research Institute. He received his B.S. in electrical engineering from the University of Belgrade in 1994. He is currently completing his M.S. degree at the University of Texas at Arlington. His research interests include robotics, real-time implementation of control systems, and discrete event systems.

Ayla Guerel received her pre-university education in Cyprus. She obtained the BS degree in 1981 at Hacettepe University, Ankara, and the MS degree in 1983 at Georgia Institute of Technology, Atlanta, both in Electrical Engineering. She received the Ph.D degree in 1989 at Bilkent University, Ankara. She was awarded a TUBITAK Scholarship for undergraduate study in Turkey, a Fulbright 
Scholarship for graduate study in the U.S. and a Fulbright Award for postgraduate research in the U.S. She was a visiting researcher during the 1997-98 academic year at the Automation and Robotics Research Institute of the University of Texas at Arlington. At present, she is with the Electrical and Electronic Engineering Department of the Eastern Mediterranean University, Famagusta, Cyprus, where she has been a member of faculty since 1983. Her research interests include modeling, simulation and control of discrete event manufacturing systems and the theory of Petri nets.

Dr. Lewis was born in Wuerzburg, Germany, subsequently studying in Chile and Scotland. He obtained the Bachelor's Degree in Physics/Electrical Engineering and the Master's of Electrical Engineering Degree at Rice University in 1971. He spent six years in the U.S. Navy, serving as Navigator aboard the frigate USS Trippe (FF-1075), and executive Officer and Acting Commanding Officer aboard USS Salinan (ATF-161). In 1977 he received the Master's of Science in Aeronautical Engineering from the University of West Florida. In 1981 he obtained the Ph.D. degree at The Georgia Institute of Technology in Atlanta, where he was employed from 1981 to 1990 and is currently an Adjunct Professor. He was awarded the Moncrief-O'Donnell Endowed Chair in 1990 at the Automation and Robotics Research Institute of The University of Texas at Arlington.

Dr. Lewis has studied the geometric properties of the Riccati equation and implicit systems; his current interests include robotics, intelligent control, neural and fuzzy systems, nonlinear systems, and manufacturing process control. He is the author/co-author of 109 journal papers, 190 refereed conference papers, five books: Optimal Control, Optimal Estimation, Applied Optimal Control and Estimation, Aircraft Control and Simulation, Control of Robot Manipulators, and the IEEE reprint volume Robot Control. Dr. Lewis is a registered Professional Engineer in the State of Texas and serves on the Editorial Board of International Journal of Control, Neural Computing and Applications, and Int. J. Intelligent Control Systems. He is the recipient of an NSF Research Initiation Grant and has been continuously funded by NSF since 1982. He received a Fulbright Research Award, the American Society of Engineering Education F.E. Terman Award, three Sigma Xi Research Awards, the UTA Halliburton Engineering Research Award, the UTA University-Wide Distinguished Research Award, the ARRI Patent Award, and the IEEE Control Systems Society Best Chapter Award (as Founding Chairman). He was selected as Engineer of the year in 1994 by the Ft. Worth IEEE Section and is a Fellow of the IEEE. He was appointed to the NAE Committee on Space Station in 1995 and to the IEEE Control Systems Society Board of Governors in 1996. 